\title{
Time Variations in Dose Rate and $\gamma$ Spectrum Measured at Tsukuba City, Ibaraki, due to the Accident of Fukushima Daiichi Nuclear Power Station
}

\author{
Toshiya SANAMI ${ }^{1, *}$, Shinichi SASAKI ${ }^{1}$, Kazuhiko IIJIMA ${ }^{1}$, \\ Yuji KISHIMOTO $^{1}$ and Kiwamu SAITO ${ }^{1}$ \\ ${ }^{1}$ Applied Physics Laboratory, High Energy Accelerator Research Organization, 1-1 Oho, Tsukuba-shi, Ibaraki 305-0801, Japan
}

\begin{abstract}
The time variations in the dose rate and $\gamma$ spectrum of radioactive nuclides originating from the Accident of Fukushima Daiichi Nuclear Power Station were measured at Tsukuba City, Ibaraki, during the period from 15th March to 9th April 2011. The radiation dose peaked three times during the period from 15 th to 16 th March $(1.27 \mu \mathrm{Sv} / \mathrm{h}$ at maximum). The contribution of Xe-133 to the dose rate was observed in the $\gamma$ spectrum obtained from the $5 \mathrm{~h}$ measurement during the peaks on 15th and 16th March, indicating that radioactive plume passed through Tsukuba City at that time. After the peaks, a dose rate increase with rainfall was observed on 21th March, dominating the integral dose rate measured at Tsukuba City. The dose after the rainfall comes from I-131, Cs-134, and Cs-137 that can be observed as peaks in the spectra.
\end{abstract}

KEYWORDS: variation in dose rate, NaI scintillator, Tsukuba City, Ibaraki, radioactive plume, Fukushima Daiichi Nuclear Power Station

\section{Introduction}

Due to an earthquake in the Pacific off the coast of Tohoku, which occurred at 14:56 on March 11, 2011 and the ensuing tsunami, Fukushima Daiichi Nuclear Power Station fell into a state of station blackout ${ }^{1}$. As a result, the nuclear reactors lost coolant and fuel rods were exposed, causing a hydrogen explosion. A significant amount of radioactive materials was released. The range of dispersion of these materials and the nuclide distribution were thought to be influenced by the conditions of release, wind direction, surrounding landscape, weather, etc. It was necessary to clarify the conditions under which measurements were taken and to compile the measured data regarding the radiation dose and radioactivity, such as ambient dose equivalent and fallout composition over a wide-ranging area and over different time spans.

The High Energy Accelerator Research Organization in Oho, Tsukuba-City, Ibaraki-Prefecture focused on the necessity of acquiring such data and started continuous measurement of dose rate and pulse-height spectrum using a $\mathrm{NaI}(\mathrm{Tl})$ scintillation detector (hereinafter $\mathrm{NaI}$

* Corresponding author, E-mail: toshiya.sanami@kek.jp

DOI : 10.15669 /fukushimainsights. Vol.4.11

(C) 2021 Atomic Energy Society of Japan. All rights reserved.

Originally published in Transactions of the Atomic Energy Society of Japan (ISSN 1347-2879), Vol. 10, No. 3, p.163-169

(2011) in Japanese. (Japanese version accepted: May 25, 2011) 
detector) starting at 14:13 on March 14 . The dose rate abruptly increased three times before dawn on March 15 and another increase in dose was observed on March 21 due to rainfall. The variation in the dose rate of $\gamma$-rays over time and the time-dependent change of the pulse-height spectrum measured using the NaI detector are summarized in this paper. Based on the data, the characteristics of the dose rate increase and residual radionuclides were discussed.

\section{Measurement Methodology}

\section{Measurement Location}

The measurement location was inside a prefabricated house (for radiation monitoring at the site boundary) at the High Energy Accelerator Research Organization in Oho, Tsukuba-City, Ibaraki-Prefecture (located $165 \mathrm{~km}$ from Fukushima Daiichi Nuclear Power Station by straightline distance, $36^{\circ} 08^{\prime} 50^{\prime \prime} \mathrm{N} 140^{\circ} 04^{\prime} 40^{\prime \prime} \mathrm{E}$ ). The monitoring house is shown in Figure 1 and the layout is depicted schematically in Figure 2. The house was placed in a wood near an asphalt paved road (20 m wide). The distance from the site boundary was $20 \mathrm{~m}$ and the distance from the asphalt paved road was $5 \mathrm{~m}$. The floor area of the house was $5.2 \times 2.3 \mathrm{~m}$ and the height was $2.1 \mathrm{~m}$. The floors, walls, and the roof were made of plywood and steel sheet. The house had two windows of $90 \times 170 \mathrm{~cm}$ each, and a ventilation fan. The ventilation fan operated continuously until a power outage on March 22. The installation location of the NaI detector is shown in the enlarged drawing in Figure 2. The NaI detector was installed at a height of $120 \mathrm{~cm}$ from the floor and $6 \mathrm{~cm}$ away from the window.

The dose rate was measured at a point inside the monitoring house and at a location outside the house using a $\mathrm{NaI}(\mathrm{Tl})$ scintillation survey meter at the time of installation and at the times when an increase in dose rate occurred. The doses inside were approximately the same as those outside; the dose rate level was confirmed for both conditions.

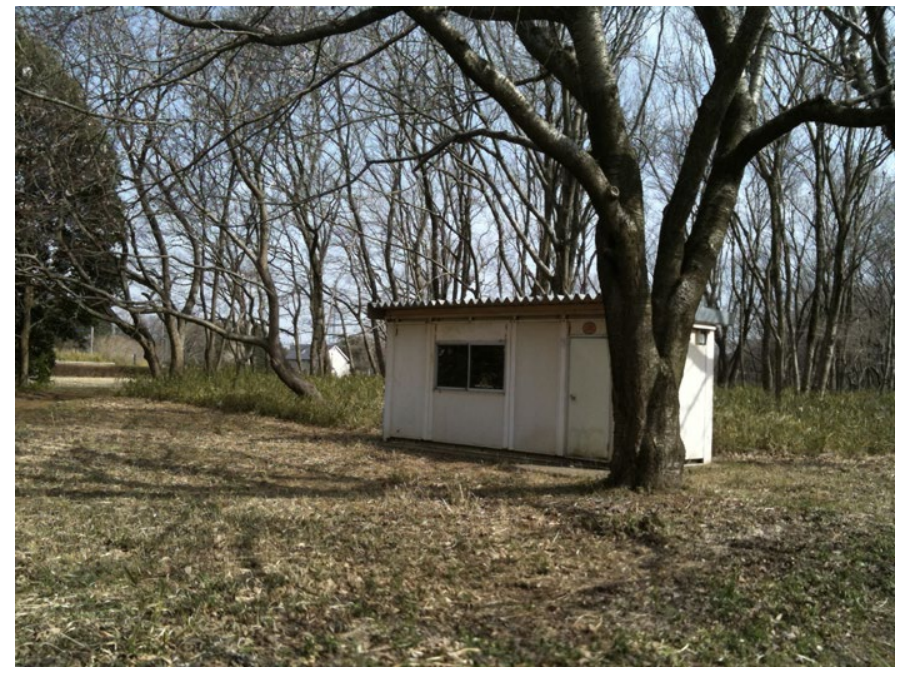

Figure 1 Picture of a monitoring house

The house was located at the High Energy Accelerator Research Organization at $36^{\circ} 08^{\prime} 50^{\prime \prime} \mathrm{N}$, $140^{\circ} 04^{\prime} 40^{\prime \prime} \mathrm{E}, 165 \mathrm{~km}$ south of the Fukushima-Daiichi Nuclear Power Station. A monitoring house containing a $\mathrm{NaI}$ scintillation detector was placed in a copse at the site. 


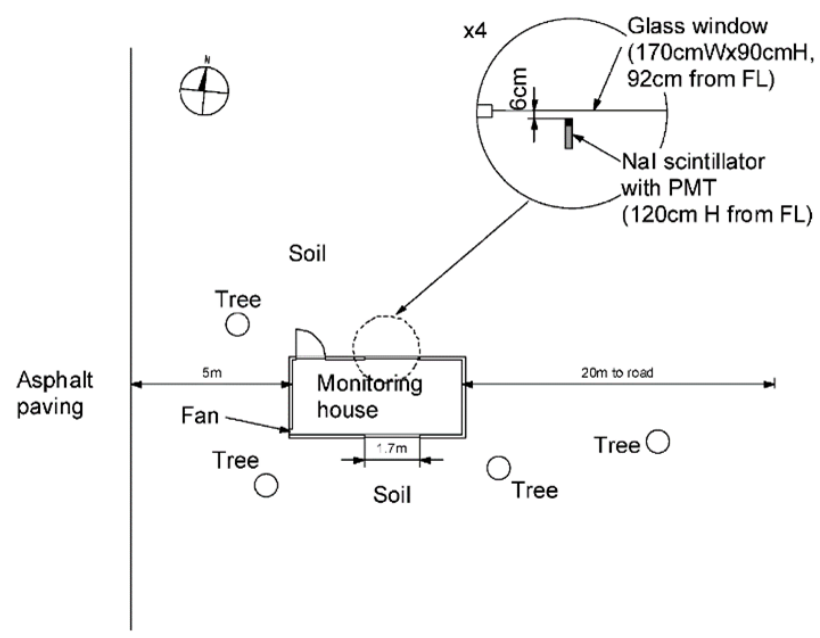

Figure 2 Plan view of the monitoring house

The house was placed on ground consisting of soil. The NaI scintillation detector was placed $120 \mathrm{~cm}$ above the floor, and $6 \mathrm{~cm}$ away from a north-facing window.

\section{NaI Detector and Data Recording System}

The $\mathrm{NaI}$ detector (Bicron, model 2M2/2) had a NaI(Tl) crystal ( 2 in. diameter $\times 2$ in. thick) mounted on the photomultiplier tube. The crystal was covered by a $0.02 \mathrm{in}$. thick aluminum casing coated with white reflective material. The output of the photomultiplier was amplified via a pre-amplifier and a main amplifier. The events having a pulse-height greater than a $\gamma$-ray energy of $118 \mathrm{keV}$ were selected using a single-channel analyzer. Counts of these pulse signals were recorded every 10 seconds and continuously written to a compact flash card. The output of the main amplifier, pulse height, was analyzed using a multi-channel pulse-height analyzer, and the pulse-height spectrum of $\gamma$-rays was acquired continuously, with measurement restart every 5 hours. Pulse-count data were recorded throughout the period from 14:57 on March 14 until 0:00 on April 9. The pulse-height spectrum data were acquired during that entire period excluding March 20 through March 22 and again from March 29 through March 31.

\section{Derivation of Dose Rate}

The time-dependent variation in dose rate was estimated based on the pulse-count data obtained by the $\mathrm{NaI}$ detector. The compensation of energy dependence in the detection efficiency based on the pulse-height signal should be applied to dose measurements using a NaI(Tl) scintillator, but this method was not available during this time because only part of the pulse-height distribution was acquired.

Furthermore, under these measuring conditions, it was likely that the $\gamma$-rays from the existing/adhered radionuclides in/on (1) the atmosphere, (2) the soil surface, and (3) roof, walls, windows, etc. of the prefabricated house were measured, so it was difficult to define the detection efficiency because the intensity and energy distribution fluctuated over time. Hence, the pulse-count rate was converted to a dose rate by standardizing to the measurement of a $1 \mathrm{~cm}$ dose equivalent on the $\mathrm{NaI}(\mathrm{Tl})$ scintillation survey meter implemented with an energy compensation circuit.

As a standard, a NaI(Tl) scintillation survey meter (Hitachi Aloka Medical Ltd., TCS-171, 
hereinafter $\mathrm{NaI}$ survey meter) was used. A NaI detector and a $\mathrm{NaI}$ survey meter were placed in the same position and the time constant was set to 30 seconds. The survey meter readings were recorded. The pulse-count rate of the $\mathrm{NaI}$ detector was normalized to this value. The normalization factor was $5.55 \times 10^{-4} \mu \mathrm{Sv} / \mathrm{h} / \mathrm{cps}$. It was assumed that this normalization factor could vary with fluctuations in the $\gamma$-ray energy distribution in the field. Hence, regarding the spectra that were acquired separately from the count rate and accumulated for 5 hours, a comparison was made on the $1 \mathrm{~cm}$ dose equivalent rate derived using the $\mathrm{G}$ function ${ }^{2)}$ of the 2 in. diameter $\times$ 2 in. thick NaI scintillation detector. The dose rate based on the above normalization factor was consistent with the value derived by the $\mathrm{NaI}$ survey meter within a margin of $10 \%$. Based on this, the dose rate during the measurement period was determined from the pulse-signal count rate, acquired over the entire period, using this normalization factor.

\section{Measured Results}

\section{Time-Variation of Dose Rate}

The time-valiation of dose rate is shown in Figure 3. The lines in the above figure show the dose rate converted from the count rate of the $\mathrm{NaI}$ detector and the dots indicate the measurement
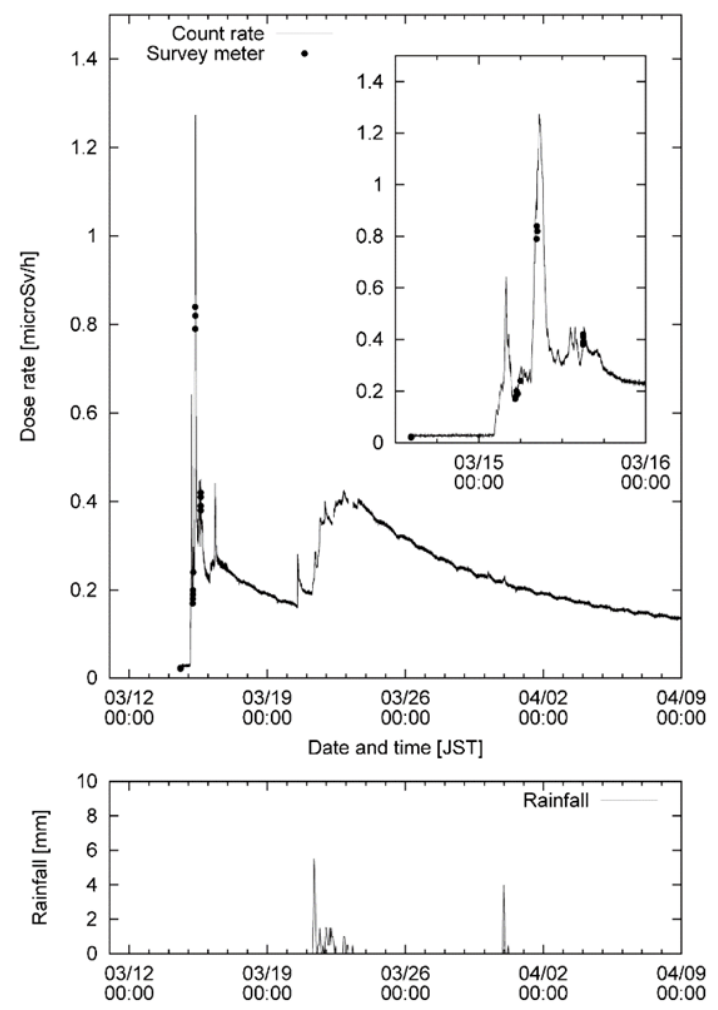

Figure 3 (Above) Dose rate for the entire period (from Mar. 12 to Apr. 9) and its increase from Mar. 14 to Mar. 16. The line shows the count rate above the threshold (118 keV equivalent) of the NaI scintillation detector. The points represent dose rate measured by a NaI scintillation survey meter with an energy compensation circuit. The count rate is normalized to the dose rate of the survey meter.

(Below) Rainfall data during the period near the site (Simotsuma area) 
by the NaI survey meter made at the same time. The two measurements are consistent with each other both in terms of the period of dose rate peaks and other periods having different pulseheight distributions. It was assumed that the variations in the pulse-height spectrum during this period did not seriously influence the conversion.

It is understood from these charts that the first arrival of the radioactive plume at Tsukuba-City, Ibaraki-Prefecture was at 2:13 on March 15. The first peak was $0.64 \mu \mathrm{Sv} / \mathrm{h}$ at 3:56 on March 15, the second was $1.27 \mu \mathrm{Sv} / \mathrm{h}$ at 8:42 on the same day. On the next day, March 16, a peak of $0.44 \mu \mathrm{Sv} / \mathrm{h}$ was observed at 8:38, and after that the dose monotonically decreased until the rate rise on March 20. In the lower chart of Figure 3, the precipitation data are shown at AMEDAS located at Nihongi, Shimotsuma-City, Ibaraki-Prefecture, which is the nearest one from the measuring point ${ }^{3}$. Rain was observed to fall from March 21 through 23 and on March 26, and a significant rise in the dose rate was seen on March 21. An increase in the dose rate, presumably due to rainfall, was observed after a small peak of $0.27 \mu \mathrm{Sv} / \mathrm{h}$ was observed at 13:36 on March 20, and the dose rate increased to $0.36 \mu \mathrm{Sv} / \mathrm{h}$ at 16:53 on March 21. The missing dose rate data during this period was caused by a power outage due to the electrical leakage that occurred at the monitoring house. The peak component on March 15 and that on March 21 were markedly different in terms of the time variation of the dose rate. While the peak component on March 15 showed a rapid increase and decrease in the dose rate, that on March 21 showed a slow increase and decrease in the dose rate. Accordingly, the increase in the dose rate was attributed to the radionuclides that were deposited near the monitoring house. Consequently, that increase in the dose rate dominated the dose rate changes after that and the cumulative dose at that point. Additionally, rainfall was observed at midnight on March 30, but no obvious increase in the dose rate was observed. Based on this observation, it was inferred that there was no significant increase in atmospheric radioactivity after the rain that fell on March 21.

It is important to understand the origin of the radioactivity on March 21 for radiological protection by elucidating the mechanism by which the dose rate increased due to rainfall.

\section{Time Variation of Pulse-height Spectrum}

Typical pulse-height spectra are shown together with the time-variation of the dose rate in Figures 4-10. The upper part in each chart shows the time-variation of the dose rate, and the marks on the graph indicate the start and stop time of the pulse-height spectrum shown in the lower part.

In the upper panel, the enlarged chart of the time-variation of the dose rate from March 15 through March 17 shows detail of the period. The measuring energy range of the pulse-height spectrum was $3 \mathrm{MeV}$, but to make the peaks easier to recognize, the range higher than $2 \mathrm{MeV}$ was omitted in Figures 4-9.

Figure 4 shows the pulse-height spectrum measured for approximately 4 hours. It shows the first increase of dose. Although there is a continuous spectrum due to various $\gamma$-ray peaks overlapping, the typical $\gamma$-ray energies for major nuclides are shown in this figure. The nuclides were chosen based on short half-life components that were deduced from the long half-life nuclides observed on the pulse-height spectrum after decay-out, the measured data on fallout substances in the Tsukuba area ${ }^{4)}$, and, the data measured by Fukushima-Prefecture ${ }^{5)}$. This is discussed later in the paper.

Figure 5 shows the pulse-height spectrum measured somewhat later than the spectrum shown in Figure 4. The time period was 5 hours from 15:15 until 20:19 on March 15. For comparison, the spectrum in Figure 4 is also shown. It can be seen from this chart that in this period, the first two increases in dose had already ended and the contribution of comparatively short half-life 

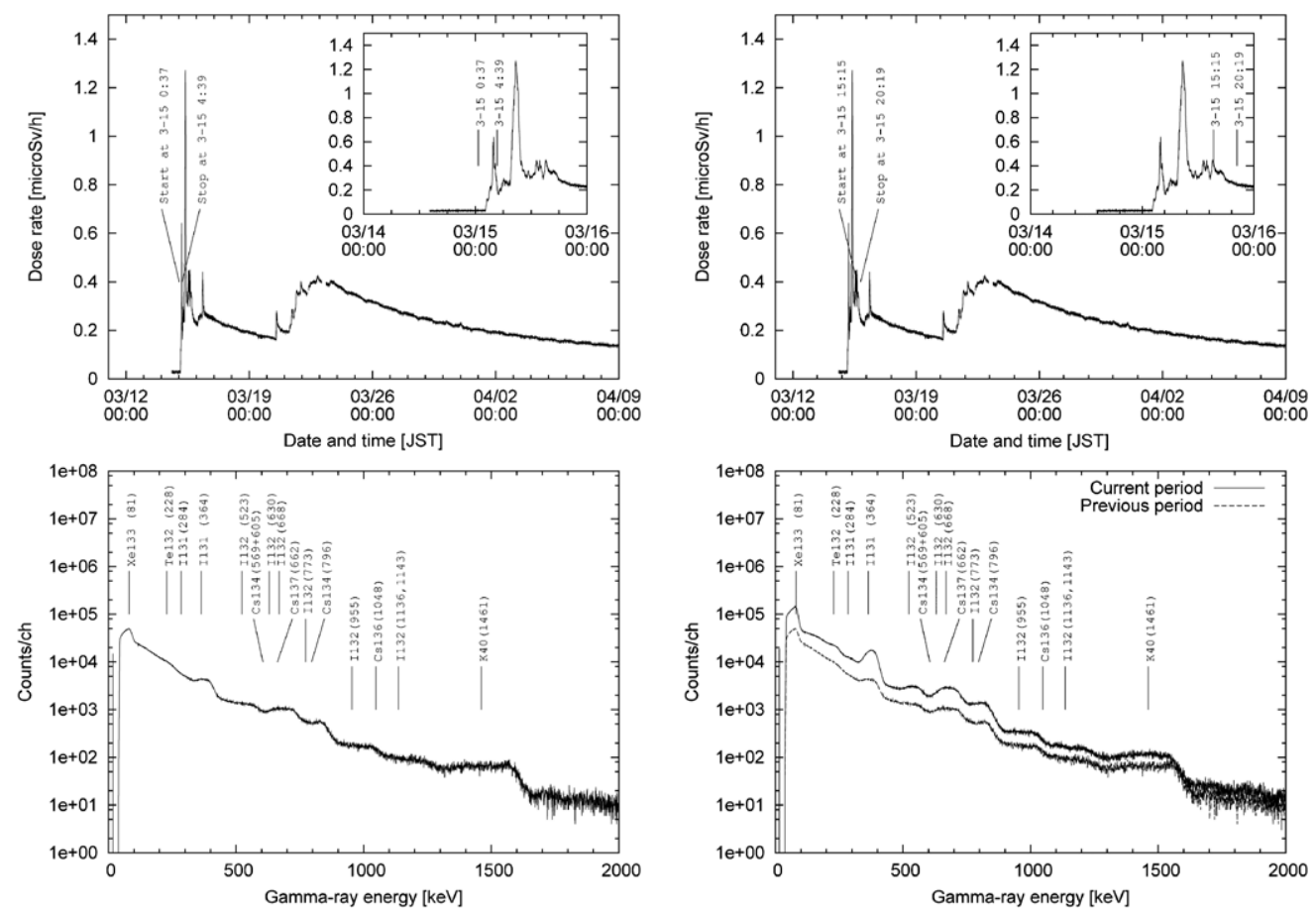

Figure 4 (Above) Dose rate for the entire period and its increase from Mar. 14 to Mar. 16. The period for the energy spectrum shown is indicated by the two lines with date and time.

(Below) Energy spectrum for the first period (from 3-15 0:37 to 3-15 4:39) The period contains the first dose rate peak due to the accident at the Fukushima Daiichi Nuclear Power Station.

The energies of major peaks from isotopes identified by other reports are indicated on the spectrum.

Figure 5 (Above) Dose rate for the entire period and its increase from Mar. 14 to Mar. 16. The period contains several small dose rate peaks after the largest peak.

(Below) Energy spectrum for the second period (from 3-15 15:15 to 3-15 20:19) in comparison with the first period Several broad peaks were observed because many $\gamma$ emissions of various energy levels contributed to the spectrum.

nuclides such as Xe-133 and Te-132 generally decreased. The main peak at $364 \mathrm{keV}$ of I-131 can be clearly seen for this reason.

Figure 6 shows the pulse-height spectrum measured from 20:19 on March 15 until 1:22 on March 16, immediately before the dose increase. The complete disappearance of Xe-133 can be seen. If the half-life of 5.243 days for Xe-133 is also considered, it becomes apparent that the variation in the dose rate was caused by the onward migration of Xe-133. An independent increase in Xe-133 is observed in Figure 7. The pulse-height spectrum more than 5 hours before the peak dose on March 16 indicates that it may be observed before the dose rise.

Figure 8 shows the pulse-height spectrum after the rise in dose on March 16. The comparison in the lower chart is the pulse-height spectrum in the period starting from 6:26 and ending at 11:29 on March 16, which includes the rise in dose on March 16. The rapid decrease of both Xe-133 and a $\gamma$-ray component from I-132, having various energy peaks, were clearly observed. Xe-133 was therefore considered a good marker for the instantaneous increase in dose. 

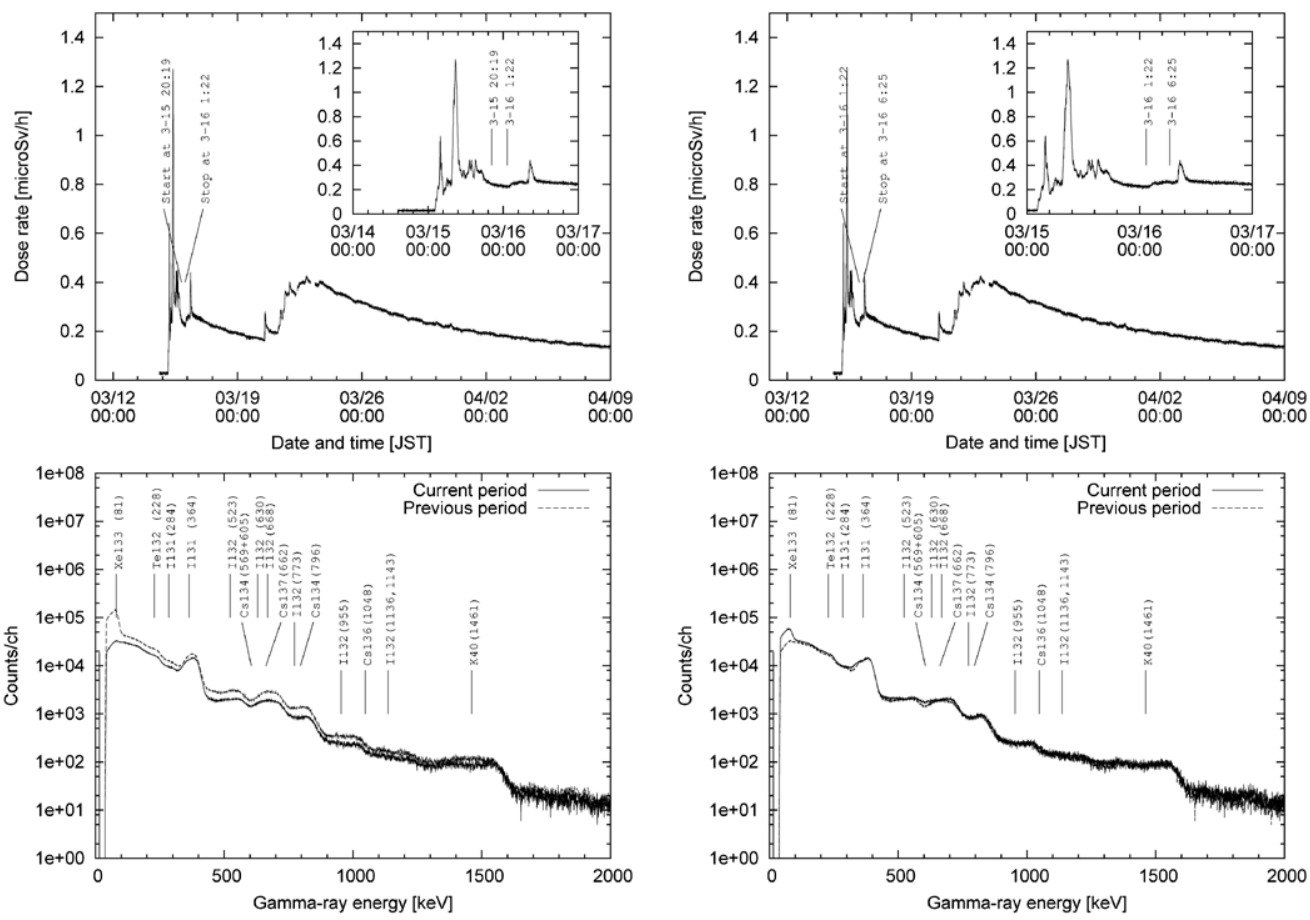

Figure 6 (Above) Dose rate over the entire period and its increase from Mar. 14 to Mar. 17. The period contains no dose rate peak. (Below) Energy spectrum for the third period (from 3-15 20:19 to 3-16 1:22) in comparison with the second period. The contribution from Xe-133 completely disappeared after the peaks.

Figure 7 (Above) Dose rate over the entire period and its increase from Mar. 15 to Mar. 17. The dose rate in the period increases slightly without any peak.

(Below) Energy spectrum for the fourth period (from 3-16 1:22 to 3-16 6:25) in comparison with the third period. The difference between the two spectra is entirely due to the contribution from $\mathrm{Xe}-133$, because Xe-133, an inert gas, can travel freely with the atmosphere.

Figure 9 shows the pulse-height spectrum (from 19:19 on March 23 until 0:21 on March 24) after the rain that fell on March 21. For the purpose of comparison, the pulse-height spectrum before the rain (from 11:04 on March 19 until 16:05 on March 19) is shown with the pulseheight spectrum after the rain. There was no obvious change in the shape of the spectrum before and after the rain. The rise in the dose rate after March 21 was clearly understandable as different from the rise in dose rate due to the radioactive plume accompanied by Xe-133. However, the rise in dose caused by the radioactive plume was only temporary as can be understood from the time-dependent fluctuations in the dose rate, but the duration of the rise in dose due to rain was long, and as a result, large cumulative values were acquired. The dose rate as of April 9 was inferred as being mainly due to the rain that fell on March 21.

Figure 10 shows the comparison of the pulse-height spectrum from 21:47 on March 31 until 2:49 on April 1 and the spectrum from 16:46 on March 31 through 21:47 on March 31, the measurement period immediately before it. Over this time period, there was no significant change in the pulse-height spectrum, instead the absolute value gradually decreased. The observed 

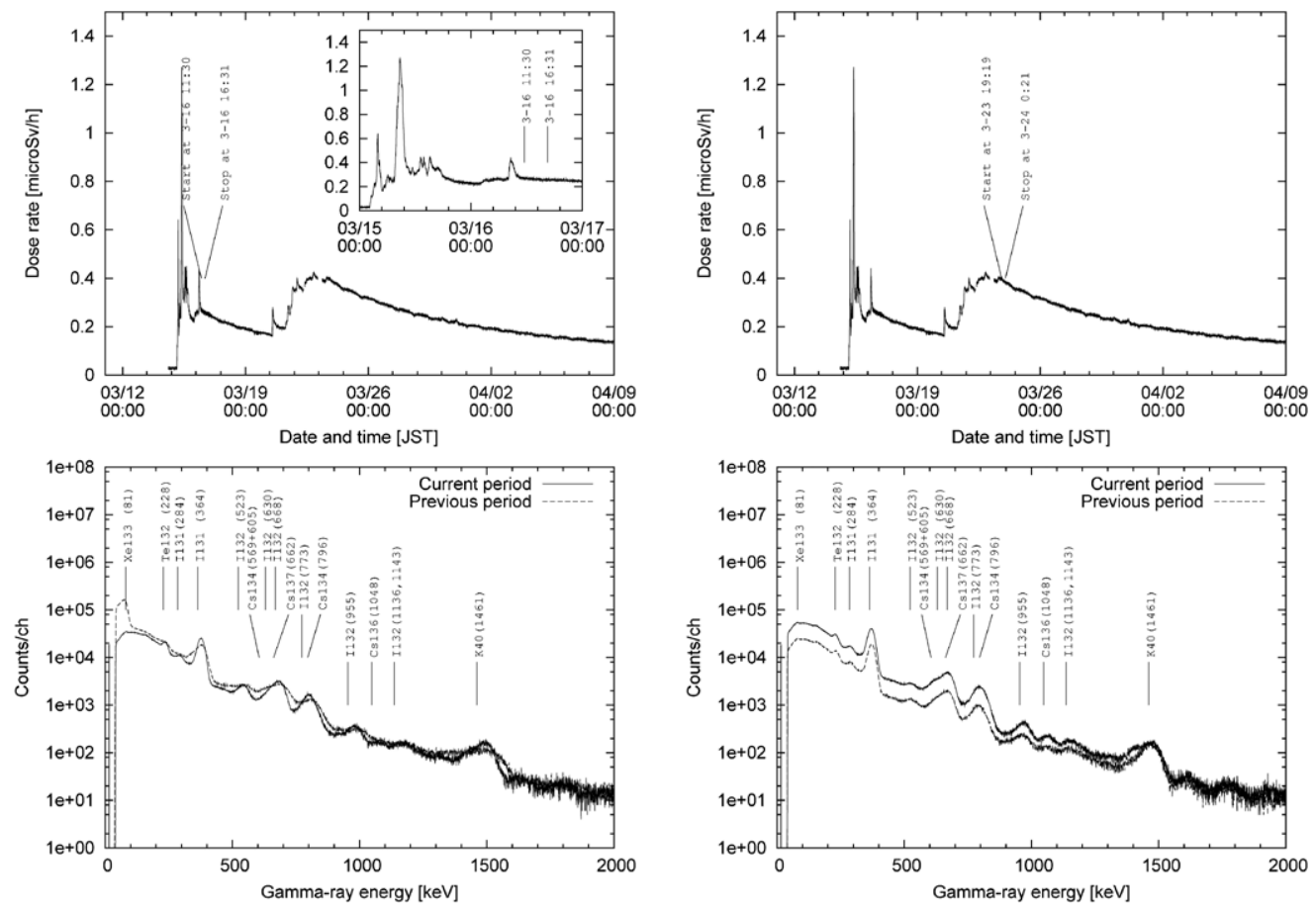

Figure 8 (Above) Dose rate over the entire period and its increase from Mar. 15 to Mar. 17.

This period corresponds to that after the dose rate peak on Mar. 16.

(Below) Energy spectrum for the sixth period (from 3-16 11:30 to 3-16 16:31) in comparison with fifth period (from 3-16 6:26 to $3-1611: 29)$

Contribution from Xe-133 has completely disappeared.

Figure 9 (Above) Dose rate over the entire period (Below) Energy spectrum for the 18th period (from 3-23 19:19 to 3-24 0:21) in comparison with 17 th period (from 3-19 11:04 to 3-19 16:05)

The 18th period corresponds to the time after rainfall and the 17 th refers to the time before rainfall. Both spectra have similar shapes, which indicates that the mechanism responsible for the increasing dose rate following rainfall was different from the mechanism that increased the dose rate during the plume.

nuclides over this time period were I-131, Cs-134, and Cs-137, and after this the dose rate declined according to the half-life of the nuclides.

\section{Time-Dependent Variation in Peak Components}

The time-dependent fluctuations in net yield with regard to each peak component of the pulse-height spectrum shown in Figures 4-10 are shown in Figure 11. The peak components were classified into four groups: (1) Xe-133 (energy $81 \mathrm{keV}$, branch ratio $38.4 \%$ (only values are listed in the following)), (2) I-131 (364 keV, 81.7\%), (3) I-132 (523 keV, 16.0\%, $630 \mathrm{keV}$, $13.3 \%, 668 \mathrm{keV}, 98.7 \%$ ), Cs-134 (605 keV, 97.6\%), Cs-137 (662 keV, 85.1\%), (4) I-132 (773 $\mathrm{keV}, 75.6 \%$ ), and Cs-134 (796 keV, 85.5\%), taking the measured nuclides, the energy and branch ratio of $\gamma$-rays, and the energy resolution of the $\mathrm{NaI}$ detector into consideration.

The chart of the peak yield of Xe-133, the second from the top in Figure 11, shows that Xe-133 has a high count rate during the dose rate peak period on March 15 and 16 and after that 

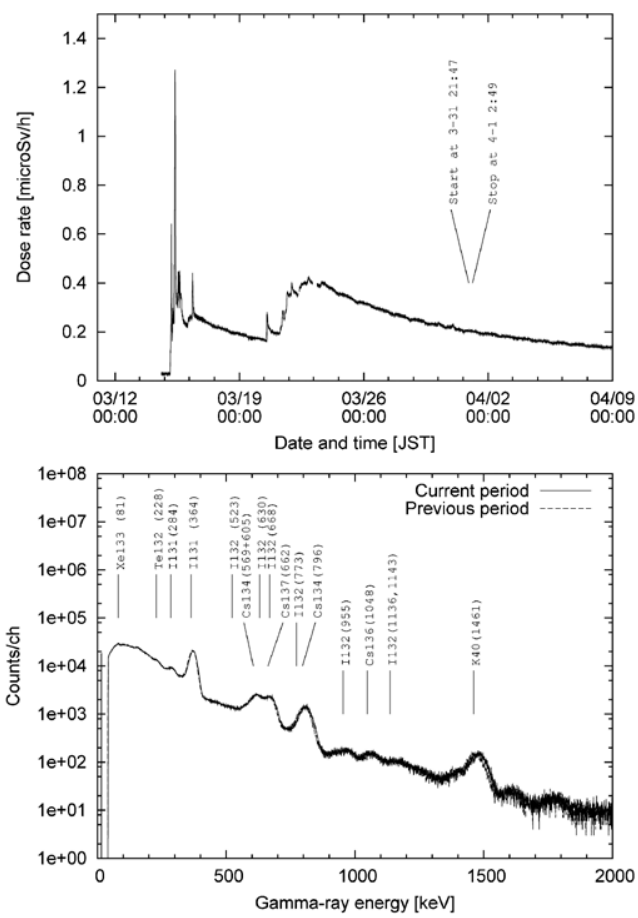

Figure 10 (Above) Dose rate over the entire period (Below) Energy spectrum for the $41 \mathrm{st}$ period (from 3-31 21:47 to 4-1 2:49) in comparison with that in the 40th period (from 3-31 16:46 to 3-31 21:47)

The 41 st spectrum is almost the same as that in the 40th period. Based on their energies, the peaks on the spectrum can be identified as $\gamma$-rays from I-131, Cs134, Cs-137, Cs-136 and K-40.
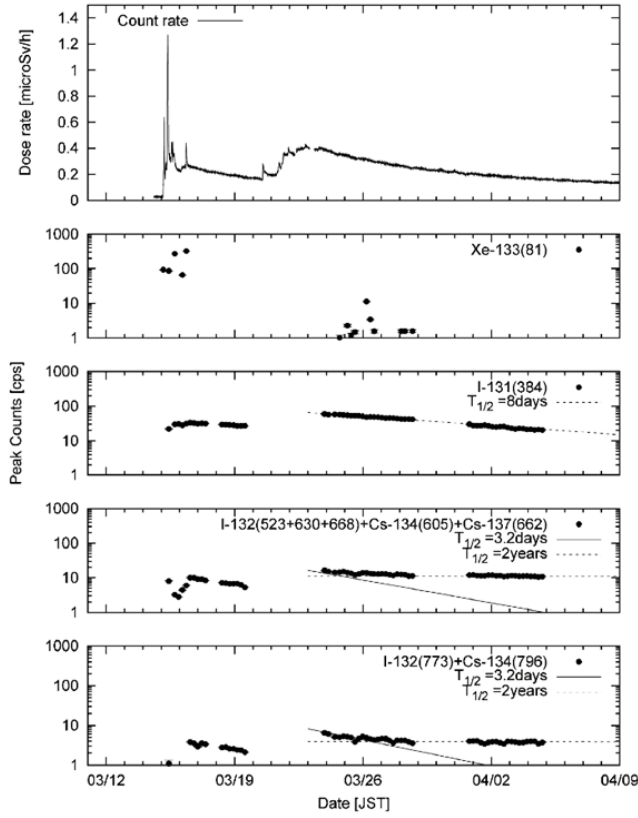

Figure 11 Relationship between dose rates over the entire period (top figure) and peak count rates for $\gamma$-rays of "Xe-133", "I-131", "I-132, Cs-134, and Cs-137", and "I-132 and Cs-134" (4 lower figures)

The line on the plot for I-131 shows the decay rate with the half-life of I-131. The solid and dotted lines on the plots of "I-132, Cs-134, and Cs-137" and "I-132 and Cs-134" correspond to the decay rates with the half-lives of Te-132 (3 days, parent of I-132) and Cs-134, respectively.

its count rate remains low. This low count rate was considered to be due to the shape of the pulseheight distributions near the peak of Xe-133 and the I-131 $\gamma$-rays. Thus, it did not indicate the real presence of Xe-133. Consequently, most the Xe-133 was measured on March 15 and 16. A small increase in the count rate on the order of several cps was measured on March 26 as well, which can be attributed to the contribution of Xe-133 based on a comparison between the pulse-height spectra during the period before and after, as shown in Figure 12.

The third chart from the top in Figure 11 shows the time-dependent fluctuation of the peak yield of I-131. Because it shows a higher count rate compared to other peak components after the increase in dose on March 21, it was assumed that it was responsible for a major portion of the dose rate. Because the count rate was a greater than $60 \mathrm{cps}$ on March 23, the contribution was expected to be less than that of $\mathrm{Cs}$, as shown below in late April after three half-life periods. The dotted line in the chart shows the decrease in count rate with a half-life of 8 days. The peak count rate was consistent with this dotted line.

The fourth and fifth charts from the top in Figure 11 show the peak count rates of Cs-134, 137, and I-132. Cs and I were not separated in either of the groups due to the energy resolution 


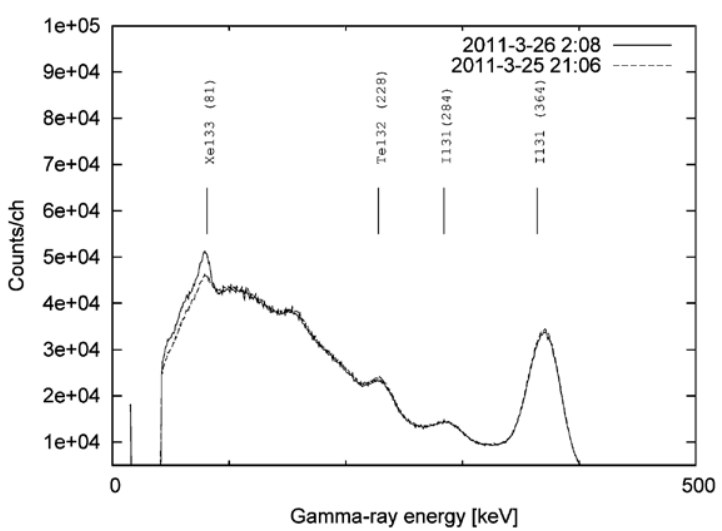

Figure $12 \mathrm{NaI}$ detector pulse-height spectrum for 5-hour periods starting at 2:08 on Mar. 26 and 21:06 on Mar. 25

The difference between the two spectra indicates the arrival of Xe-133, which suggests that additional, smaller amounts of gas were being released from the nuclear power station.

of the NaI detector. The solid line in the chart shows the decrease in count rate for a half-life of 3.2 days (equivalent to the half-life of the parent nuclide, Te-132 of I-132) and the dotted line shows that for a half-life of 2 years (equivalent to the half-life of Cs-134). The peak count rates in the chart are consistent with the half-life line of 3.2 days before March 28 and the half-life line of 2 years after March 28. Based on this, it was understood that I-132 was dominant during the period until March 28, while Cs was dominant after March 28. Furthermore, the radioactivity ratio was estimated based on the peak yields of Cs-137 and Cs-134 using the peak detection efficiency of the 2 in. diameter $\times 2$ in. thick $\mathrm{NaI}$ scintillator when a point source estimated by EGS5 ${ }^{6}$ ) was assumed. The ratio of Cs-137 to Cs-134 was 1:1.15 based on the data in the fourth and fifth charts of Figure 11 for the period after I-132 decayed. The uncertainty of this value was approximately $5 \%$, excluding the factors due to the distribution of radio active nuclides. In the future, a more precise ratio of Cs-137 to Cs-134 will be obtained by measuring the distribution for radionuclides adhering to the soil, structures, etc. around the measuring point.

\section{Conclusions}

The measurement of $\gamma$-rays derived from the Fukushima Daiichi Nuclear Power Station was conducted using a NaI scintillation detector in Tsukuba-City, Ibaraki-Prefecture, and the time-variation of dose rate and pulse-height spectra were measured. During the measurement period, the increase in dose rate due to the radioactive plume in the early stage and later as a result of rainfall were observed, and the different decay behavior of the two were clarified. Based on the pulse-height spectrum during the time when the radioactive plume passed over the city in the early stage, it was clarified that the plume contained a large quantity of Xe-133. Xe-133 was observed on March 26 as well. The pulse-height spectrum before and after the increase in dose due to rain that fell on March 21 did not show a significant difference, and it was found that I-131, Cs-134, and Cs-137 were the major components. The radioactivity ratio of Cs-134 to $\mathrm{Cs}-137$ was estimated to be $1: 1.15$.

We express our gratitude to Mrs. Takeshi Machida, Kenichi Yokota of JREC, who kindly cooperated in the calibration of detectors. 


\section{References}

1) Nuclear and Industrial Safety Agency, Conditions of Fukushima Dai-ichi Nuclear Power Station Unit 1-6 (As of 6:00 April 30th, 2011), News release, Ministry of Economy, Trade and Industry (2011). [in Japanese]

2) M. Tsutsumi, K. Saito, S. Moriguchi, Spectrum-dose Conversion Operators, G(E) Functions of NaI(Tl) Scintillators Adapted for Effective Dose Equivalent Quantities, JAERI-M 91-204. [in Japanese]

3) Japan Meteorological Agency, http://www.data.jma.go.jp/obd/stats/etrn/index.php. [in Japanese]

4) Environmental radiation at High energy accelerator research organization, http://www.kek.jp/quake/ radmonitor. [in Japanese]

5) H. Matsumura, K. Saito, J. Ishioka et al., "Behavior of radioactive materials from Fukushima Daiichi Nuclear Power Station obtained by radiation scan on the expressways," Trans. At. Energy Soc. Jpn., $10[3]$, to be published. [in Japanese]

6) H. Hirayama, Y. Namito, A. F. Bielajew et al., The EGS5 Code System, SLAC-R-730 (2005) and KEK Report 2005-8 (2005). 